\title{
Histochemical comparison of the epithelial mucins in the ileum in Crohn's disease and in normal controls
}

\author{
C. F. A. CUlling, P. E. Reid, W. L. DUNN, AND M. G. ClAY \\ From the Department of Pathology and Department of Surgery, Faculty of Medicine, University of British \\ Columbia, Vancouver, Canada V6T 1W5
}

SUMMARY A comparison of routine and special histochemical methods that were applied to the epithelial mucins of small intestine from patients with Crohn's disease and from normal controls showed that the normal small intestine (ileum) goblet cells secrete a predominantly non-sulphated sialomucin and that, in contradistinction to the colon, the neuraminidase insensitivity of the sialic acids of the small intestine was not due to either $\mathrm{O}$-acylation at $\mathrm{C}_{4}$ or an ester substituent at $\mathrm{C}_{1}$. Presumably this implies that the protection against enzyme attack afforded to the mucosa by the mucin coat in the small intestine utilises a different mechanism from that in the colon and that, although in many respects the small intestinal mucins in Crohn's disease, and in normal controls are similar, there is an increase in side-chain O-acylated sialic acids in such mucins in Crohn's disease. This difference has not been described before, probably because it can be seen only after staining such sections by the PAT/KOH/PAS and the PBT/KOH/PAS techniques.

In contrast to the extensive investigation of the histochemical properties of the epithelial mucins of the normal and diseased human large intestine (Lev and Spicer, 1965; Lev and Orlic, 1974; Greco et al., 1967; Hellstrom and Fisher, 1967; Hradsky et al., 1967; Goldman and Ming, 1968; Filipe, 1969, 1971, 1972; Gad, 1969a, b; Johansen and Kay, 1969; Filipe and Dawson, 1970; Korhonen et al., 1971; Makela et al., 1971; Subbuswamy, 1971a, b; Filipe and Branfoot, 1974) there have been few detailed studies of the epithelial mucins of the normal small intestine (Lev and Spicer, 1965; Goldman and Ming, 1968; Lev, 1968; Gad, 1969a, b; Subbuswamy, 1971a; Lev et al., 1972) and only a small number of observations of any changes that may occur in Crohn's disease (Gad, 1969b; Lev and Spicer, 1965). In fact, Dawson (1972) stated: 'There is little reliable work on mucins in the small bowel in Crohn's disease'.

In a series of histochemical investigations (Culling et al., 1971, 1974a, b, 1975, 1976; Reid et al., 1973, $1974,1976)$ we have described new methods for the examination of epithelial mucins of the gastrointestinal tract and, in particular, for the location of

Received for publication 26 April 1977
O-acyl substituents on the side chains of sialic acid residues. We now report the results obtained from the application of some of these methods, together with standard procedures, to the epithelial mucins of normal human small intestine and of small intestine from cases of Crohn's disease.

\section{Material and methods}

Selected samples of human small intestine were obtained, with the exception of two normal intestines taken at necropsy, from the surgical pathology files of the Vancouver General Hospital. The following tissues were examined: (1) sixteen specimens, each of $2.5 \mathrm{~cm}$ long, were taken at $10-\mathrm{cm}$ intervals along $200 \mathrm{~cm}$ of small intestine starting at the ileocaecal valve; (2) five specimens, each $2.5 \mathrm{~cm}$ long, were taken at $2 \cdot 5-\mathrm{cm}$ intervals along the terminal ileum; and (3) nine specimens, each $2.5 \mathrm{~cm}$ long, were taken at $7 \cdot 5-\mathrm{cm}$ intervals along the first $90 \mathrm{~cm}$ of each of two small intestines taken at necropsy followed by a further four specimens, each $2.5 \mathrm{~cm}$ long, taken at about $180,270,360$, and $450 \mathrm{~cm}$ from the ileocaecal valve.

Thirty-five specimens were examined from seven cases of Crohn's disease. Two of them showed 1063 
severe ulceration and sloughing of the mucosa together with associated inflammatory changes, two showed moderate to severe changes, and three were moderate in nature. Judged from the degree of fibrosis all were cases of longstanding disease. All specimens were fixed in $10 \%$ formol calcium. They were processed through alcohol and chloroform and embedded in paraplast embedding medium. Sections were cut at 5 microns.

\section{HISTOCHEMICAL TECHNIQUES}

All standard procedures were carried out as described by Culling (1974).

Saponification $(\mathrm{KOH})$. Sections were treated at room temperature in $0.5 \%$ potassium hydroxide in $70 \%$ ethanol for 30 minutes.

Acid hydrolysis. Sections were heated with $0 \cdot 1 \mathrm{~mol} / 1$ sulphuric acid at $80^{\circ} \mathrm{C}$ for 1 hour. Both the sections and the acid were preheated to $80^{\circ}$ before starting hydrolysis (Culling et al., 1974b). Control sections were incubated in water alone.

Neuraminidase digestion. Sections were incubated for 24 hours at $37^{\circ} \mathrm{C}$ with Vibrio cholerae neuraminidase (Behringwerke, protease, aldolase, and lecithinase- $\mathrm{C}$ free) 100 units $/ \mathrm{ml}$ in $0.05 \mathrm{M}$ acetate buffer $\mathrm{pH}$ 5.5 containing $0.1 \%$ calcium chloride. Control sections were incubated in buffer alone.

STAINING TECHNIQUES

The following staining techniques were used.

Periodic acid Schiff $(P A S)$ to demonstrate periodate reactive vicinol diols.

Periodic acid-phenylhydrazine-Schiff (PAPS) (Spicer, 1961) Acid groups on or adjacent to residues containing aldehydes generated by periodate oxidation will prevent phenylhydrazine blockade of subsequent Schiff staining.

Periodate-borohydride technique/saponification/PAS (PBT/KOH/PAS) (Reid et al., 1973; Culling, 1974) To demonstrate the $\mathrm{KOH} / \mathrm{PAS}$ effect in isolation; this effect has been associated with side-chain O-acylated sialic acids (Culling et al., 1974b).

Periodate-thionin Schiff/saponification/PAS (PAT/ $K O H / P A S)$ (Culling et al., 1976) To demonstrate pre-existing tissue vicinal diols in blue with a thionin Schiff reagent, and subsequently $\mathrm{KOH} / \mathrm{PAS}$ positive material in red (by saponification followed by the standard PAS technique).

Mixed diamine (Spicer, 1965) to demonstrate those acid groups which are ionised at or below the pH $(3 \cdot 5-4 \cdot 0)$ of the dye bath.

Periodic acid-mixed diamine (Spicer, 1965). Abolition or reduction of mixed diamine staining by prior periodate oxidation indicates the presence of periodate-sensitive vicinal diols close to acid groups.
Alcian blue $0.3 \%$ at pH 2.5 to demonstrate those acid groups in mucins which remain ionised at or below pH $2 \cdot 5\left(\mathrm{COOH}, \mathrm{OSO}_{3} \mathrm{H}\right)$.

Alcian blue $0.3 \%$ at $\mathrm{pH} 1.0$ to demonstrate those acid groups in mucins which remain ionised at or below $\mathrm{pH} 1.0\left(\mathrm{OSO}_{3} \mathrm{H}\right)$.

\section{Results}

NORMAL HUMAN SMALL INTESTINE

The intensity of the PAS staining of the epithelial mucins was much diminished by the interpolation of a phenylhydrazine blockade (PAPS procedure, Spicer, 1961) between the periodate oxidation step and staining with Schiff's reagent. Probably, therefore, most of the PAS reactivity of the mucins was due to vicinal diols which were neither on nor near to residues bearing anionic groups. Therefore much of the PAS reactivity was not due to sialic acids but probably to neutral hexoses within the carbohydrate chains of the mucin.

All residues carrying ionic groups apparently either possessed or were near periodate sensitive vicinal diols, since mixed diamine staining was abolished by prior periodate oxidation. Presumably these anionic groups were carboxyls since there was little staining with alcian blue at $\mathrm{pH} 1 \cdot 0$, although the mucins stained strongly with alcian blue at $\mathrm{pH}$ $2 \cdot 5$. Selected samples stained with high iron diamine (Spicer, 1965) gave similar results. These results are consistent with the presence of sialomucins.

The $\mathrm{KOH} / \mathrm{PAS}$ effect as seen in the PAT/KOH/ PAS and PBT/KOH/PAS procedures was very much weaker than that we have observed in the large intestine (Reid et al., 1973; Culling et al., 1974a, 1975, 1976). Moreover, it was apparently confined to the final two feet $(53 \mathrm{~cm})$ of the ileum. In large intestine mucins the $\mathrm{KOH} / \mathrm{PAS}$ effect has been associated with the de-O-acylation of side-chain O-acylated sialic acids (Culling et al., 1974a, 1976).

To investigate the sialic acids of the small intestine epithelial mucins we compared the intensity of the staining of control sections with alcian blue at $\mathrm{pH}$ 2.5 and that of sections which had been subjected to various procedures. These were (1) neuraminidase digestion; or (2) saponification followed by neuraminidase digestion; or (3) hydrolysis with $0.1 \mathrm{~mol} / 1$ sulphuric acid at $80^{\circ} \mathrm{C}$; or (4) saponification followed by the hydrolysis procedure; or (5) saponification alone. These procedures have been used previously (Culling et al., 1974a, b; Reid et al., 1976) to identify 4-O-acyl sialic acids.

Alcianophilia was only ever slightly, and then inconsistently, diminished by neuraminidase digestion. This enzymic removal of sialic acid was often slightly increased by prior saponification, but at no 
time were large quantities of sialic acid removed.

Acid hydrolysis alone completely abolished alcianophilia in a section from one of the two intestines removed at necropsy and considerably reduced it in sections from all other specimens.

When acid hydrolysis was preceded by saponification there was a further reduction in alcian blue staining in all sections. In the other small intestine removed at necropsy staining was completely abolished. These results indicated that alcianophilia in all specimens was almost completely due to sialic acid residues. The reason for the apparent differences between necropsy specimens and between them and the surgical specimens is presumed to be due to post-mortem changes.

Acid hydrolysis (alone or after saponification) was much more effective in decreasing the alcianophilia of the epithelial mucins than either of the neuraminidase treatments. Since saponification alone had no effect on the intensity of staining with alcian blue we concluded that the resistance of the mucins to digestion with neuraminidase was not a consequence of ester substituents at either position C4 or position $\mathrm{Cl}$ of the sialic acid residues (Culling et al., 1974a; Reid et al., 1976).

Acid hydrolysis or saponification followed by acid hydrolysis had little or no effect on the PAS staining of the epithelial mucins. Since sialic acids without side-chain substituents are PAS positive this would indicate that the hydrolytic removal of such sialic acids had generated new vicinal diols.

In previous studies of colonic mucins (Culling et al., 1974a) we have shown that the $\mathrm{KOH} / \mathrm{PAS}$ effect is associated with ester substituents on sialic acid side-chains. This was established by showing that acid hydrolysis or neuraminidase digestion decreased PAS staining in saponified sections. In view of the above results it was not possible to demonstrate this relationship in normal small intestine mucins.

\section{CROHN'S DISEASE}

In general the staining properties of the small intestine epithelial mucins in cases of Crohn's disease were similar to those of normal small intestine, except that in almost all $(97 \%)$ of the sections examined the KOH/PAS effect, as shown by the PBT/KOH/PAS technique, was stronger than that in normal terminal ileum-an impression confirmed by the intensity of the red staining in the PAT/KOH/ PAS procedure.

The severity of the disease seemed generally but not invariably to be associated with the more pronounced increase in the KOH/PAS effect, which gave a red-purple colour with the PAT/KOH/PAS technique. The areas of mucosa which appeared histologically to be relatively normal, present in some sections, did not obviously show a predisease type of change.

Acid hydrolysis after saponification caused a small but consistent decrease in PAS staining which was greater than that caused by acid hydrolysis alone. That was in direct contrast to normal small intestinal mucin. Presumably this indicates that the $\mathrm{KOH} / \mathrm{PAS}$ effect seen was, at least in part, associated with sialic acid residues.

There was little or no evidence for the presence of O-sulphate esters and staining with alcian blue at pH 1.0 was absent in most $(81 \%)$ of the sections examined.

\section{Discussion}

Previous studies of normal adult and fetal small intestine (Lev and Spicer, 1965; Goldman and Ming, 1968; Lev, 1968; Gad, 1969a; Subbuswamy, 1971a; Lev et al., 1972) indicated that the goblet cells contained an acid, non-sulphated mucin. The basophilia of the mucins was unaffected by hyaluronidase and the mucin was not completely neuraminidase labile (Lev and Spicer, 1965; Gad, 1969a). Fetal mucins were more neuraminidase-labile than those of the adult (Lev, 1968). Lev and Spicer (1965) found that saponification slightly enhanced the neuraminidase lability of the mucins, but Gad (1969a) was unable to demonstrate this effect. Periodate oxidation abolished mixed diamine staining (Lev and Spicer, 1965) while Schiff staining in the PAPS procedure was minimal (Gad, 1969a). The results we obtained with normal small intestine are in general agreement with these other investigators in that we also found a neuraminidase insensitive mucin in goblet cells and a similar staining pattern with the mixed diamine, periodate-mixed diamine, and PAPS procedures.

As we have reported previously (Culling et al., 1975), the terminal $60 \mathrm{~cm}$ of ileum exhibited the $\mathrm{KOH} / \mathrm{PAS}$ effect, which is otherwise confined to the large intestine (Culling et al., 1971, 1974a, 1975; Reid et al., 1973). In the large intestine O-acylated sialic acids have been shown to be responsible for this effect, but in this instance we were unable to demonstrate this relationship.

The abolition of nearly all the alcianophilia at pH 2.5 by acid hydrolysis sequences shows that the basophilia of the mucins was due to sialic acid residues. Such residues, however, were almost completely resistant to digestion with Vibrio cholerae neuraminidase and prior saponification resulted in only a small enhancement in their lability towards neuraminidase. This indicated that, unlike colonic mucin, the resistance to neuraminidase digestion was not a consequence of ester substituents at position 
C4 or position C1 (Culling et al., 1974a; Reid et al., 1976). The above results are unlikely to be due to fixation since studies on mouse salivary mucins (Sorvari and Lauren, 1973) indicated that formalin fixation did not affect their neuraminidase susceptibility. Chemical studies of rat intestinal mucin (Bella and Kim, 1972; Forstner et al., 1973) indicated that the sialic acids are almost completely neuraminidase labile, but similar studies apparently have not been carried out for the human glycoprotein(s). Presumably, therefore, the glycosidic linkage between the sialic acids and the remainder of the glycoprotein in the small intestinal mucins of man are largely neuraminidase resistant.

Histochemical studies of the mucins in Crohn's disease of the small intestine have been confined to a small number of specimens (Lev and Spicer, 1965; Gad, 1969b). They showed that, in contrast to normal small intestine, there was an acid-sulphated mucin present; the presence of this sulphated mucin, however, was not specific for Crohn's disease.

Our results indicated that in general the small intestinal mucins in Crohn's disease were similar to those found in normal small intestine but there was an increase in the KOH/PAS effect, and this increase, in contrast to the KOH/PAS-positive mucin in normal small intestine, could be shown to be associated with sialic acid. We did not, however, find an increase in acid-sulphated mucin as described by Lev and Spicer (1965) and Gad (1969b).

We have previously suggested (Culling et al., 1974a) that the O-acylated sialic acids in the colonic mucins are part of a protective mechanism against the action of the faecal stream and specifically against the neuraminidases secreted by the bacterial flora. Such protection would be afforded by $\mathrm{C} 4$ substitution, and presumably some is also provided by substituents at $\mathrm{C7}$ or $\mathrm{C} 8$, or both (Schauer and Faillard, 1968). It is therefore surprising to find that in the normal small intestine the sialic acid residues do not have an ester substituent at either $\mathrm{C4}$ or $\mathrm{Cl}$ but are nevertheless neuraminidase insensitive. In Crohn's disease there was an increase in sialic acids substituted at $\mathrm{C7}$ or $\mathrm{C8}$, or both (KOH/PAS effect), which were also neuraminidase insensitive. This could be an attempt to provide further protection against some other unknown factor. So far as we are aware this is the first report of a specific difference in epithelial mucins in Crohn's disease, be this cause or consequence.

We thank Mrs Odile Polo and Mrs Jana Tomanek for their skilled technical assistance and Mrs Elizabeth Barstow for secretarial assistance. The work was supported by the Medical Research Council of Canada.

\section{References}

Bella, A., Jr. and Kim, Y. S. (1972). Rat small intestinal mucin: Isolation and characterisation of a watersoluble mucin fraction. Archives of Biochemistry and Biophysics, 160, 679-689.

Culling, C. F. A. (1974). Handbook of Histopathological and Histochemical Techniques, 3rd edition. Butterworths, London.

Culling, C. F. A., Reid, P. E., Burton, J. D., and Dunn, W. L. (1975). A histochemical method of differentiating lower gastrointestinal tract mucin from other mucins in primary or metastatic tumours. Journal of Clinical Pathology, 28, 656-658.

Culling, C. F. A., Reid, P. E., Clay, M. G. and Dunn, W. L. (1974a). The histochemical demonstration of O-acylated sialic acid in gastrointestinal mucins: their association with the potassium hydroxide-periodic acid-Schiff effect. Journal of Histochemistry and Cytochemistry, 22, 826-831.

Culling, C. F. A., Reid, P. E., and Dunn, W. L. (1971). The effect of saponification upon certain histochemical reactions of the epithelial mucins of the GI tract. Journal of Histochemistry and Cytochemistry, 19, 654-662.

Culling, C. F. A., Reid, P. E., and Dunn, W. L. (1974b), A problem in the removal of sialic acid by acid hydrolysis. Stain Technology, 49, 317-318.

Culling, C. F. A., Reid, P. E., and Dunn, W. L. (1976) A new histochemical method for the identification and visualisation of both side chain acylated and non acylated sialic acids. Journal of Histochemistry and Cytochemistry, 24, 1225-1230.

Dawson, I. M. P. (1972). The histochemistry of Crohn's disease. Clinics in Gastroenterology, 1, 309-320.

Filipe, M. I. (1969). Value of histochemical reactions for mucosubstances in the diagnosis of certain pathological conditions of the colon and rectum. Gut, 10, 577-586.

Filipe, M. I. (1971). ${ }^{35}$ Sulphur uptake in the mucosa adjacent to carcinoma of the large intestine. Histochemical Journal, 3, 27-35.

Filipe, M. I. (1972). The value of a study of the mucosubstances in rectal biopsies from patients with carcinoma of the rectum and lower sigmoid in the diagnosis of premalignant mucosa. Journal of Clinical Pathology, 25, 123-128.

Filipe, M. I., and Branfoot, A. C. (1974). Abnormal patterns of mucus secretion in apparently normal mucosa of large intestine with carcinoma. Cancer, 34, 282-290.

Filipe, M. I., and Dawson, I. M. P. (1970). The diagnostic value of mucosubstances in rectal biopsies from patients with ulcerative colitis and Crohn's disease. Gut, 11, 229-234.

Forstner, J. F., Jabbal, I., and Forstner, G. G. (1973). Goblet cell mucin of rat small intestine. Chemical and physical characterization. Canadian Journal of Biochemistry, 51, 1154-1166.

Gad, A. (1969a). A histochemical study of human alimentary tract mucosubstances in health and disease. I. Normal and tumours. British Journal of Cancer, 23, 52-63. 
Gad, A. (1969b). A histochemical study of human alimentary tract mucosubstances in health and disease. II. Inflammatory conditions. British Journal of Cancer, 23, 64-68.

Goldman, H., and Ming, S. (1968). Mucins in normal and neoplastic gastrointestinal epithelium. Histochemical distribution. Archives of Pathology, 85, 580-586.

Greco, V., Lauro, G., Fabbrini, A., and Torsoli, A. (1967). Histochemistry of the colonic epithelial mucins in normal subjects and in patients with ulcerative colitis. Gut, 8, 491-496.

Hellstrom, H. R., and Fisher, E. R. (1967). Estimation of mucosal mucin as an aid in the differentiation of Crohn's disease of the colon and chronic ulcerative colitis. American Journal of Clinical Pathology, 48, 259-268.

Hradsky, M., Langr, F., Nerad, V., and Skaunic, V. (1967). Alterations of the histological and histochemical pattern in ulcerative colitis biopsy specimens. American Journal of Proctology, 18, 392-398.

Johansen, P. G., and Kay, R. (1969). Histochemistry of rectal mucus in cystic fibrosis of the pancreas. Journal of Pathology, 99, 299-306.

Korhonen, L. K., Mäkelä., V. and Lilius, G.(1971). Carbohydrate rich compounds in the colonic mucosa of man. II. Histochemical characteristics of colonic adenocarcinomas. Cancer, 27, 128-133.

Lev, R. (1968). A histochemical study of glycogen and mucin in developing human foetal epithelia. Histochemical Journal, 1, 152-165.

Lev, R., and Orlic, D. (1974). Histochemical and radioautographic studies of normal human fetal colon. Histochemistry, 39, 301-311.

Lev, R., Siegel, H. I., and Bartman, J. (1972). Histochemical studies of developing human fetal small intestine. Histochemistry, 29, 103-119.

Lev, R., and Spicer, S. S. (1965). A histochemical comparison of human epithelial mucins in normal and in hypersecretory states including pancreatic cystic fibrosis. American Journal of Pathology, 46, 23-47.

Mäkelä, V., Korhonen, L. K., and Lilius, G. (1971).
Carbohydrate-rich compounds in the colonic mucosa of man. I. Histochemical characteristics of normal and adenomatous colonic mucosa. Cancer, 27, 120-127.

Reid, P. E., Culling, C. F. A., and Dunn, W. L. (1973). Saponification-induced increase in the periodic acidSchiff reaction in the gastrointestinal tract. Mechanism and distribution of the reactive substance. Journal of Histochemistry and Cytochemistry, 21, 473-482.

Reid, P. E., Culling, C. F. A., and Dunn, W. L. (1974). The histochemical interpretation of the complex results of methylation upon gastrointestinal tract mucins with special reference to the periodic acid-Schiff reactivity. Journal of Histochemistry and Cytochemistry, 22, 986-991.

Reid, P. E., Culling, C. F. A., Dunn, W. L., and Clay, M. G. (1976). The use of a transesterification technique to distinguish between certain neuraminidase resistant epithelial resistant epithelial mucins. Histochemistry, 46, 203-207.

Schauer, R., and Faillard, H. (1968). Zur Wirkungsspezifität der Neuramidase. Das Verhalten isomerer N. O-Diacetylneuraminsäureglykoside im Submaxillarismucin von Pferd und Rind bei Einwirkung bakterieller Neuramidase. Hoppe-Seyler's Zeitschrift für Physiologische Chemie, 349, 961-968.

Sorvari, T. E., and Laurén, P. A. (1973). The effect of various fixation procedures on the digestability of sialomucins with neuraminidase. Histochemical Journal, 5, 405-412.

Spicer, S. S. (1961). The use of various cationic reagents in histochemical differentiation of mucopolysaccharides. American Journal of Clinical Pathology, 36, 393-407.

Spicer, S. S. (1965). Diamine methods for differentiating mucosubstances histochemically. Journal of Histochemistry and Cytochemistry, 13, 211-234.

Subbuswamy, S. G. (1971a). Patterns of mucin secretion in human intestinal mucosa. Journal of Anatomy, 108, 291-293.

Subbuswamy, S. G. (1971b). Mucosubstances in neoplasms of the human colon and rectum. Gut, 12, 200-207. 